\title{
Positivity of the Bondi Energy in Einstein-Maxwell Axion-dilaton Gravity
}

\author{
Marek Rogatko * \\ Technical University of Lublin \\ 20-618 Lublin, Nadbystrzycka 40, Poland \\ rogat@tytan.umcs.lublin.pl \\ rogat@akropolis.pol.lublin.pl
}

November 20, 2018

PACS numbers: 04.50.+h.

\begin{abstract}
We present a proof of the positivity of the Bondi energy in Einstein-Maxwell axion dilaton gravity, being the low-energy limit of the heterotic string theory. We consider the spacelike hypersurface which asymptotically approaches a null cone and on which the equations of the theory under consideration are given. Next, we generalize the proof allowing the hypersurface having inner boundaries.
\end{abstract}

\section{Introduction}

In the framework of general relativity, there are two distinct regimes where we can measure the mass. The first, at null infinity, yields the Bondi mass while the second, at spacial infinity, represents the Arnowitt-Desser-Misner (ADM) mass. The main difference between these two notions is that the Bondi momentum is dynamical while the ADM one is not. The Bondi quantity is tightly bounded with an instant of retarded time and changes according to the radiation which the source emits. The ADM four-momentum vector is fixed. The tantalizing question is whether the physically reasonable gravitating systems can radiate more energy than they initially have, i.e. this is a question whether the Bondi energy can become negative.

During last years there were heavy attempts to prove the positivity of the Bondi mass. In a number of recent papers this long-standing conjecture has been confirmed. Nester and Israel [1]

\footnotetext{
*Supported in part by KBN grant 2 P03B 09318.
} 
generalized the spinor method of Witten [2] and Nester [3] to present that a system satisfying the dominant energy condition has a positive total energy and a timelike four-momentum measured at spacelike or future null infinity.

Horowitz et al [4] proved that for a spacetime which was asymptotically flat at null infinity and for which the dominant energy condition held and existed a nonsingular spacelike hypersurface which asymptotically approached the null cone of constant retarded time near null infinity, the Bondi four-momentum was a future directed timelike or null vector. The Bondi four-momentum vanished iff we had a flat spacetime in the domain of dependence for the considered hypersurface. It was done by extending the Witten's proof of the positivity of energy to the case of null infinity. In [5] it was shown that neither the ADM nor the Bondi four-momentum of an isolated system in general relativity can be null.

The previous arguments presented by Schoen and Yau [6] were modified to demonstrate the positivity of the Bondi mass [7]. For a given spacelike hypersurface asymptotic to the null cone, they have constructed a three-dimensional asymptotically flat initial data set which ADM mass is not greater than the Bondi mass of the null cone under consideration. The positivity of the Bondi mass follows from the previously proved theorem [6]. Their method is very sensitive to the choice of the spacelike hypersurface asymptotic to the null cone.

Ludvigsen and Vickers [8] managed to prove positivity of the Bondi momentum by means of choosing the hypersurface which consisted a compact spacelike part and a null part in the asymptotic region and considered the projection of the Witten's type of equation on it.

Reula and Tod [9] gave a new type of the proof of the positivity of the Bondi mass. They proved the existence of solutions of Witten's equation for the case in which a spacelike slice on which the equation was given was asymptotically null. They provided the unified treatment of the ADM and Bondi mass. Among all, by a suitable choice of boundary conditions the positivity of Bondi energy was shown for the case where the hypersurface was no longer free of inner boundaries, but was bounded by the union of finite number of marginally trapped surfaces.

Horowitz and Tod [10] considered asymptotically constant solutions to the Weyl neutrino equation on an asymptotically flat spacetime. They found that there existed a collection of conserved vector fields associated with these solutions, depended on the local stress energy of the matter and which had the property that their integrals over spacelike surfaces yielded the total ADM or Bondi four-momentum of the spacetime.

Lately, in [11] the new spinorial proof applying to both the ADM and Bondi energies was achieved. In this attitude no existence theorems on noncompact hypersurfaces were needed to obtain the desire results.

The main aim of the paper is to provide some continuity with our previous works concerning 
the problem of the ADM mass and the positive mass theorem for black holes in the low-energy limit of heterotic string theory, in Einstein-Maxwell axion-dilaton (EMAD) gravity. We try to enlarge our considerations to the problem of the Bondi mass in EMAD system. The organisation of this paper is as follows. In section 2 we review the basic filed equations of the theory under consideration and prove the positivity of the Bondi mass in the case in which the spacelike hypersurface on which the equations are given is asymptotically null. We also provide the generalization of this theorem allowing the hypersurface having inner boundaries. Section 3 summarizes our results.

In our paper we shall use two-component spinor notation [13. Spinor indices are denoted by capital letters. Our signature of $g_{\mu \nu}$ is $(+---)$ and convention for the curvature tensor is $2 \nabla_{[\alpha} \nabla_{\beta]} \eta_{\gamma}=-R_{\alpha \beta \gamma \delta} \eta^{\delta}$. The Einstein equations are $G_{\mu \nu}=-T_{\mu \nu}$.

\section{Positivity of the Bondi Mass}

The so-called low-energy limit of superstring theories provides an interesting generalization of the Einstein-Maxwell (EM) theory. A simplified model of this kind in an Einstein-Maxwell axion-dilaton (EMAD) coupled system containing a metric $g_{\mu \nu}, U(1)$ vector fields $A_{\mu}$, a dilaton $\phi$ and three-index antisymmetric tensor field $H_{\alpha \beta \gamma}$. The action has the form [14

$$
I=\int d^{4} x \sqrt{-g}\left[-R+2(\nabla \phi)^{2}+\frac{1}{3} H_{\alpha \beta \gamma} H^{\alpha \beta \gamma}-e^{-2 \phi} F_{\alpha \beta} F^{\alpha \beta}\right]+I_{m a t t e r}
$$

where the strength of the gauge fields is descibed by $F_{\mu \nu}=2 \nabla_{[\mu} A_{\nu]}$ and the three index antisymmetric tensor is defined by the relation

$$
H_{\alpha \beta \gamma}=\nabla_{\alpha} B_{\beta \gamma}-A_{\alpha} F_{\beta \gamma}+\text { cyclic. }
$$

In four dimensions $H_{\alpha \beta \gamma}$ is equivalent to the Peccei-Quin pseudoscalar and implies the following:

$$
H_{\alpha \beta \gamma}=\frac{1}{2} \epsilon_{\alpha \beta \gamma \delta} e^{4 \phi} \nabla^{\delta} a .
$$

A straightforward consequence of the definition (3) is that the action (11) can be written as

$$
I=\int d^{4} x \sqrt{-g}\left[-R+2(\nabla \phi)^{2}+\frac{1}{2} e^{4 \phi}(\nabla a)^{2}-e^{-2 \phi} F_{\alpha \beta} F^{\alpha \beta}-a F_{\mu \nu} * F_{\mu \nu}\right]+I_{m a t t e r}
$$

where $* F_{\mu \nu}=\frac{1}{2} \epsilon_{\mu \nu \delta \rho} F^{\delta \rho}$.

Using the two-component spinor notation, we define a Maxwell spinor by the relation

$$
F_{m n}=\phi_{M N} \epsilon_{M^{\prime} N^{\prime}}+\bar{\phi}_{M^{\prime} N^{\prime}} \epsilon_{M N},
$$


where

$$
\phi_{A B}=\frac{1}{2} F_{A B C^{\prime}} C^{\prime}, \quad \bar{\phi}_{A^{\prime} B^{\prime}}=\frac{1}{2} F_{C}{ }_{A^{\prime} B^{\prime}} .
$$

Then equations of motion are given by

$$
\begin{aligned}
\nabla_{M}{ }^{N^{\prime}} \phi^{M N} & =\nabla^{N}{ }_{M^{\prime}} \bar{\phi}^{M^{\prime} N^{\prime}}, \\
\nabla_{M}{ }^{N^{\prime}}\left(z \phi^{M N}\right)-\nabla^{N}{ }_{M^{\prime}}\left(\bar{z} \bar{\phi}^{M^{\prime} N^{\prime}}\right) & \left.=\mathcal{J}^{N^{\prime} N} \text { (matter }\right), \\
\nabla_{\mu} \nabla^{\mu} \phi-\frac{1}{2} e^{4 \phi}(\nabla a)^{2}-e^{2 \phi}\left(\phi_{M N} \phi^{M N}+\bar{\phi}_{M^{\prime} N^{\prime}} \bar{\phi}^{M^{\prime} N^{\prime}}\right) & =0, \\
\nabla_{\mu} \nabla^{\mu} a+4 \nabla_{\mu} \phi \nabla^{\mu} a+2 i e^{-4 \phi}\left(\bar{\phi}_{M^{\prime} N^{\prime}} \bar{\phi}^{M^{\prime} N^{\prime}}-\phi_{M N} \phi^{M N}\right) & =0, \\
6 \Lambda \epsilon_{A B} \epsilon_{A^{\prime} B^{\prime}}+2 \Phi_{A B A^{\prime} B^{\prime}}=T_{A A^{\prime} B B^{\prime}}, &
\end{aligned}
$$

where we have introduced a complex axi-dilaton $z=a+i e^{-2 \phi}$, while $\Lambda=\frac{R}{24}$ and $\Phi_{A B A^{\prime} B^{\prime}}$ is the curvature spinor (sometimes called Ricci spinor). The energy momentum tensor reads

$$
\begin{aligned}
T_{\mu \nu}(F, \phi, a)=8 e^{-2 \phi} \phi_{M N} \bar{\phi}_{M^{\prime} N^{\prime}} & -\epsilon_{M N} \epsilon_{M^{\prime} N^{\prime}}\left[2(\nabla \phi)^{2}+\frac{1}{2} e^{4 \phi}(\nabla a)^{2}\right] \\
& +4 \nabla_{M M^{\prime}} \phi \nabla_{N N^{\prime}} \phi+e^{4 \phi} \nabla_{M M^{\prime}} a \nabla_{N N^{\prime}} a
\end{aligned}
$$

To begin with we define the supercovariant derivatives for two-component spinors $\left(\alpha^{A}{ }_{(i)}, \beta_{A^{\prime}(i)}\right)$. Their forms followed easily from the adequate definition in the case of four-component spinors introduced in [12, 14], namely

$$
\begin{aligned}
\hat{\nabla}_{M M^{\prime}} \alpha_{K(i)} & =\nabla_{M M^{\prime}} \alpha_{K(i)}+\frac{i}{2} e^{2 \phi} \nabla_{M M^{\prime}} a \alpha_{K(i)}+\sqrt{2} e^{-\phi} \phi_{K M} a_{(i j)} \beta_{M^{\prime}}^{(j)}, \\
\hat{\nabla}_{M M^{\prime}} \beta_{A^{\prime}(i)} & =\nabla_{M M^{\prime}} \beta_{A^{\prime}(i)}+\frac{i}{2} e^{2 \phi} \nabla_{M M^{\prime}} a \beta_{A^{\prime}(i)}-\sqrt{2} e^{-\phi} \bar{\phi}_{A^{\prime} M^{\prime}} a_{(i j)} \alpha_{M}^{(j)},
\end{aligned}
$$

where $a_{(i j)}$ is the two-dimensional equivalent of the matrices introduced in [15].

In what follows it will be convenient to define the following quantities:

$$
\begin{aligned}
\delta \lambda_{A^{\prime}(i)}^{(\alpha)} & =\sqrt{2}\left(\sqrt{2} \nabla_{A^{\prime} B} \phi \alpha^{B}{ }_{(i)}+\frac{i}{\sqrt{2}} e^{2 \phi} \nabla_{A^{\prime} B} a \alpha^{B}{ }_{(i)}-4 i e^{-\phi} \bar{\phi}_{A^{\prime}} C^{\prime} a_{(i j)} \beta_{C^{\prime}}{ }^{(j)}\right) \\
\delta \lambda_{A(i)}^{(\beta)} & =\sqrt{2}\left(\sqrt{2} \nabla_{A}{ }^{B^{\prime}} \phi \beta_{B^{\prime}(i)}+\frac{i}{\sqrt{2}} e^{2 \phi} \nabla_{A}{ }^{B^{\prime}} a \beta_{B^{\prime}(i)}+4 i e^{-\phi} \phi_{A C} a_{(i j)} \alpha^{C(j)}\right) .
\end{aligned}
$$

The motivation for introducing these quantities is twofold (see e.g. [16]). First, we would like to achieve the desired mass bound and therefore the specific factors appear in these definitions. Second, expressions (15 - 16) have motivation as the supergravity transformation laws of the appropriate particles in the associated supergravity model. The EMAD gravity model constitutes the bosonic part of $N=4, d=4$ supergravity (the so-called $S U(4)$ version) with one gauge field [14. 
We shall consider first an everywhere spacelike, asymptotically null slice $\Sigma$ (a surface without boundary) of an asymptotically flat spacetime. Let $t^{a}$ be the unit normal to the hypersurface $\Sigma$, let $h_{a b}=g_{a b}-t_{a} t_{b}$ be the metric induced on the hypersurface $\Sigma$ and $D_{a}=h_{a}{ }^{b} \nabla_{b}$, where $\nabla_{b}$ is the covariant derivative on $M$ with respect to the metric $g_{a b}$ on $M$. Now it is easily seen that the Witten's equations on the hypersurface $\Sigma$ may be written in the form

$$
\begin{aligned}
& \hat{D}_{A A^{\prime}} \beta^{A^{\prime}{ }_{(i)}}=D_{A A^{\prime}} \beta^{A^{\prime}}{ }_{(i)}+\frac{i}{2} e^{2 \phi} D_{A A^{\prime}} a \beta^{A^{\prime}}{ }_{(i)}+\sqrt{2} e^{-\phi} t_{A A^{\prime}} t^{M M^{\prime}} \bar{\phi}^{A^{\prime}}{ }_{M^{\prime}} a_{(i j)} \alpha_{M}{ }^{(j)}, \\
& \hat{D}_{A A^{\prime}} \alpha^{A}{ }_{(i)}=D_{A A^{\prime}} \alpha^{A}{ }_{(i)}+\frac{i}{2} e^{2 \phi} D_{A A^{\prime}} a \alpha^{A}{ }_{(i)}-\sqrt{2} e^{-\phi} t_{A A^{\prime}} t^{M M^{\prime}} \phi^{A}{ }_{M} a_{(i j)} \beta_{M^{\prime}}{ }^{(j)}
\end{aligned}
$$

Let us introduce the future directed null vector $\xi_{m}$ for arbitrary (smooth) spinor fields $\left(\alpha^{M}{ }_{(i)}, \beta_{M^{\prime}(i)}\right)$ on an asymptotically flat spacetime as follows:

$$
\xi_{m}=\alpha_{M}^{(i)} \bar{\alpha}_{M^{\prime}(i)}+\bar{\beta}_{M}^{(i)} \beta_{M^{\prime}(i)}
$$

Consider next, the vector field $p_{a}$ defined by the relation [10]

$$
p_{n}=\hat{\nabla}^{m} \hat{\nabla}_{[n} \xi_{m]}
$$

From (20), one can deduce that $p_{n}$ is the divergence free, and that conserved quantity obtained by integration over the hypersurface $\Sigma$ may be expressed as an integral over asymptotic two sphere $S$. Consequently, we have

$$
\int_{\Sigma} d \Sigma_{a} p^{a}=\int_{S} d S^{n} t^{m} \hat{\nabla}_{[n} \xi_{m]}
$$

Taking into account that $\xi_{m}$ is divergence free due to the generalized Weyl equations, and using the definition of $\hat{D}_{a}$ on a hypersurface $\Sigma$ with the unit normal vector $t^{a}$, i.e.

$$
\hat{\nabla}_{a}=\hat{D}_{a}+t_{a} t^{b} \hat{\nabla}_{b}
$$

one finds that

$$
\begin{aligned}
t^{A A^{\prime}} \hat{\nabla}_{b} \alpha_{A(i)} \hat{\nabla}^{b} \bar{\alpha}_{A^{\prime}}{ }^{(i)} & =t^{A A^{\prime}} \hat{D}_{b} \alpha_{A(i)} \hat{D}^{b} \bar{\alpha}_{A^{\prime}}{ }^{(i)}+2 t^{A A^{\prime}} \hat{D}_{A^{\prime} B} \alpha^{B}{ }_{(i)} \hat{D}_{A B^{\prime}} \bar{\alpha}^{B^{\prime}(i)} \\
t^{A A^{\prime}} \hat{\nabla}_{b} \bar{\beta}_{A(i)} \hat{\nabla}^{b} \beta_{A^{\prime}}{ }^{(i)} & =t^{A A^{\prime}} \hat{D}_{b} \bar{\beta}_{A(i)} \hat{D}^{b} \beta_{A^{\prime}}{ }^{(i)}+2 t^{A A^{\prime}} \hat{D}_{A^{\prime} B} \bar{\beta}^{B}{ }_{(i)} \hat{D}_{A B^{\prime}} \beta^{B^{\prime}(i)}
\end{aligned}
$$

After a fairly lenghty calculations one can show

$$
\begin{aligned}
\frac{1}{2} \int_{S} d S^{a b} \hat{\nabla}_{a} \xi_{b} & =\frac{1}{2} \int_{\Sigma} d \Sigma T_{a b}(\text { matter }) t^{a} \xi^{b}+ \\
& -\int_{\Sigma} d \Sigma\left(t^{A A^{\prime}} \hat{D}_{b} \alpha_{A(i)} \hat{D}^{b} \bar{\alpha}_{A^{\prime}}(i)+2 t^{A A^{\prime}} \hat{D}_{A^{\prime} B} \alpha^{B}{ }_{(i)} \hat{D}_{A B^{\prime}} \bar{\alpha}^{B^{\prime}(i)}\right)
\end{aligned}
$$




$$
\begin{aligned}
& -\int_{\Sigma} d \Sigma\left(t^{A A^{\prime}} \hat{D}_{b} \bar{\beta}_{A(i)} \hat{D}^{b} \beta_{A^{\prime}}^{(i)}+2 t^{A A^{\prime}} \hat{D}_{A^{\prime} B} \bar{\beta}^{B}{ }_{(i)} \hat{D}_{A B^{\prime}} \beta^{B^{\prime}(i)}\right) \\
& +\int_{\Sigma} d \Sigma \mathcal{K}_{A A^{\prime}} t^{A A^{\prime}} \\
& +\int_{\Sigma} d \Sigma\left[\left(\delta \lambda_{A^{\prime}(i)}^{(\beta)}\right)^{\dagger} \delta \lambda_{A}^{(\beta)(i)}+\left(\delta \lambda_{A(i)}^{(\alpha)}\right)^{\dagger} \delta \lambda_{A^{\prime}}^{(\alpha)(i)}\right] t^{A A^{\prime}} \\
& -\frac{1}{\sqrt{2}} \int_{\Sigma} d \Sigma\left[J_{m}(F, \phi) t^{m}\left(\bar{\alpha}^{B^{\prime}(i)} \beta_{B^{\prime}(i)}\right)-\bar{J}_{m}(F, \phi) t^{m}\left(\alpha^{B(i)} \bar{\beta}_{B(i)}\right)\right] .
\end{aligned}
$$

The energy momentum tensor $T_{a b}($ matter $)$ yields

$$
T_{a b}(\text { matter })=T_{a b}(\text { total })-T_{a b}(F, \phi, a),
$$

while a complex current is defined as follows:

$$
J_{A A^{\prime}}(F, \phi)=\nabla_{A^{\prime} B}\left(e^{-\phi} \phi_{A}^{B}\right)
$$

The quantity $\mathcal{K}_{A A^{\prime}}$ is given by

$$
\begin{aligned}
\mathcal{K}_{A A^{\prime}} & =\frac{i e^{2 \phi}}{2} \nabla_{A B^{\prime}} \phi \nabla_{B A^{\prime}} a \xi^{B B^{\prime}}+i e^{2 \phi} \nabla_{A^{\prime} M} \phi \nabla_{A M^{\prime}} a\left(\alpha^{M}{ }_{(i)} \bar{\alpha}^{M^{\prime}(i)}-\bar{\beta}^{M}{ }_{(i)} \beta^{M^{\prime}(i)}\right) \\
& +8 \sqrt{2} i e^{-\phi} \nabla_{A^{\prime} B} \phi \phi_{C A} \bar{\beta}^{(B(i)} \alpha^{C)}{ }_{(i)}+4 \sqrt{2} e^{\phi} \nabla_{A^{\prime} B} a \phi_{A C} \bar{\beta}^{[B(i)} \alpha^{C]}{ }_{(i)} \\
& + \text { complex conjugate. }
\end{aligned}
$$

We assume that the matter energy-momentum tensor obeys the following condition:

$$
T_{a b}(\text { matter }) t^{a} \xi^{b} \geq \sqrt{2}\left[J_{m}(F, \phi) t^{m}\left(\bar{\alpha}^{B^{\prime}(i)} \beta_{B^{\prime}(i)}\right)-\bar{J}_{m}(F, \phi) t^{m}\left(\alpha^{B(i)} \bar{\beta}_{B(i)}\right)\right] .
$$

Condition (28) is stronger than the dominant energy condition ussually assummed in general relativity in order to prove the positivity of the Bondi mass. This kind of requirement is also known in Einstein-Maxwell gravity [17, 23] where it constitutes the key point of the proof. Roughly speaking relation (28) tells us that the local energy density is greater or equal to the local densities of the adequate charge densities.

Moreover we put additional conditions, namely

$$
\delta \lambda_{A(i)}^{(\alpha)}=\delta \lambda_{A^{\prime}(i)}^{(\alpha)}=\delta \lambda_{A(i)}^{(\beta)}=\delta \lambda_{A^{\prime}(i)}^{(\beta)}=0,
$$

and

$$
\mathcal{K}_{A A^{\prime}}=0
$$

The relation (29) is motivated by the invariance of the entire system under the supersymmetry transformations written in the language of two-component spinors. The other introduces relations amongs the fields in the considered theory. Relations like (30) arise in various kinds of 
the extensions of Einstein-Maxwell theory to the case of the low-energy string theory, both in proofs of the positivity of the ADM mass and the positivity of black holes masses [16, 12].

Thus one can see from the analysis of the right side of the relation (24) that taking into account (28-30) the right-hand side is positive if the third and fifth term are equal to zero. We remark that the second and the fourth terms are automatically positive since the metric on the hypersurface $\Sigma$ is negative. However, the third and the fifth term are negative, so we equate them to zero, i.e. one requires the following:

$$
\hat{D}_{A^{\prime} A^{A}} \alpha_{(i)}^{A}=0, \quad \hat{D}_{A^{\prime} A} \bar{\beta}_{(i)}^{A}=0 .
$$

There are elliptic first order equations on $\alpha^{A}{ }_{(i)}$ and $\bar{\beta}^{A}{ }_{(i)}$.

Under the conditions (28-30) one can ensure the positivity of the right-hand side of equation (24) if we find asymptotically constant solutions to (31) on the hypersurface $\Sigma$. In Einstein's gravity the existence theorem for such solutions was given by Tod and Reula in [9].

Following this method, for readers' convenience, we quote the main steps of the proof of the existence theorem for (31). Now, let define the Hilbert space [18] $\mathcal{H}$ as the completion of $C_{0}^{\infty}$ (smooth and compactly supported) $\sigma^{A}(i)$ spinor fields on $\Sigma$, under the following norm:

$$
\left(\left\|\sigma_{(i)}^{A}\right\|_{\mathcal{H}}\right)^{2}=\int_{\Sigma} d \Sigma \hat{D}_{B A^{\prime}} \sigma^{B}{ }_{(i)} \hat{D}_{A B^{\prime}} \bar{\sigma}^{B^{\prime}}{ }_{(i)} t^{A A^{\prime}}
$$

Using the generalized Hardy lemma [9] one finds that elements of $\mathcal{H}$ are measurable fields and if $\left\{\sigma^{A}{ }_{(i) m}\right\}$ are $C_{0}^{\infty}$ Cauchy sequences converging to $\sigma_{(i)}^{A}$, then $\hat{D}_{A A^{\prime}}\left(\sigma^{A}{ }_{(i) m}\right)$ weakly converges to the distributional derivative $\hat{D}_{A A^{\prime}} \sigma_{(i)}^{A}$ of $\sigma^{A}{ }_{(i) m}$. The Hardy lemma in our case can be obtained by the method presented in [9] when we change ordinary differential operator for supercovariant one acting on the hypersurface $\Sigma$. Thus, the integral (32) makes sence for all elements of $\mathcal{H}$. We shall seek the solutions of (31) in the forms

$$
\begin{aligned}
\alpha_{(i)}^{A} & =\alpha_{\infty(i)}^{A}+a_{(i)}^{A}, & a_{(i)}^{A} \in \mathcal{H}, \\
\bar{\beta}_{(i)}^{A} & =\bar{\beta}_{\infty(i)}^{A}+b_{(i)}^{A}, & b_{(i)}^{A} \in \mathcal{H},
\end{aligned}
$$

By means of the Riesz representation theorem (see e.g. 19]) one defines linear functionals

$$
\begin{aligned}
& f_{1}\left(\sigma_{(i)}^{A}\right)=-\int_{\Sigma} d \Sigma \hat{D}_{A B^{\prime}} \bar{\sigma}^{B^{\prime}{ }_{(i)}} \hat{D}_{B A^{\prime}} \alpha_{\infty(i)}^{B} t^{A A^{\prime}}, \\
& f_{2}\left(\sigma^{A}{ }_{(i)}\right)=-\int_{\Sigma} d \Sigma \hat{D}_{A B^{\prime}} \bar{\sigma}^{B^{\prime}}{ }_{(i)} \hat{D}_{B A^{\prime}} \bar{\beta}_{\infty(i)}^{B} t^{A A^{\prime}},
\end{aligned}
$$

Furthermore, using the Bondi coordinates [20] and the asymptotic values of spin coefficients found by Exton et al [21], having in mind the attitude of Bramson [22], one can assert that 
that $\alpha_{\infty(i)}^{B}$ and $\bar{\beta}_{\infty(i)}^{B}$ are asymptotically constant spinor fields. Considering, next, the CauchySchwartz inequality for spinors, one finds out that $f_{1}\left(\sigma^{A}{ }_{(i)}\right)$ and $f_{2}\left(\sigma^{A}{ }_{(i)}\right)$ are bounded linear functionals on the Hilbert space. It leads to the conclusion that there exist such $a^{A}{ }_{(i)}$ and $b^{A}{ }_{(i)}$ that

$$
\begin{aligned}
& f_{1}\left(\sigma^{A}{ }_{(i)}\right)=\int_{\Sigma} d \Sigma \hat{D}_{A B^{\prime}} \bar{\sigma}^{B^{\prime}}{ }_{(i)} \hat{D}_{B A^{\prime}} a^{B}{ }_{(i)} t^{A A^{\prime}}, \\
& f_{2}\left(\sigma^{A}{ }_{(i)}\right)=\int_{\Sigma} d \Sigma \hat{D}_{A B^{\prime}} \bar{\sigma}^{B^{\prime}}{ }_{(i)} \hat{D}_{B A^{\prime}} b^{B}{ }_{(i)} t^{A A^{\prime}} .
\end{aligned}
$$

Now, applying (34) and (35), integrating by parts we obtain

$$
\begin{aligned}
& \int_{\Sigma} d \Sigma \hat{D}_{A^{\prime}}{ }^{B} \hat{D}_{B B^{\prime}} \bar{\sigma}^{B^{\prime}}{ }_{(i)}\left(\alpha_{A \infty(i)}+a_{A(i)}\right) t^{A A^{\prime}}=0, \\
& \int_{\Sigma} d \Sigma \hat{D}_{A^{\prime}}{ }^{B} \hat{D}_{B B^{\prime}} \bar{\sigma}^{B^{\prime}}{ }_{(i)}\left(\bar{\beta}^{A \infty(i)}+b_{A(i)}\right) t^{A A^{\prime}}=0,
\end{aligned}
$$

for all $\sigma^{A}{ }_{(i)} \in C_{0}^{\infty}$. Thus, there exist $a^{A}{ }_{(i)}$ and $b^{A}{ }_{(i)}$ given by the relations (49) satisfying the weak form of equations

$$
\hat{D}_{A}{ }^{B^{\prime}} \hat{D}_{B B^{\prime}} \alpha^{A}{ }_{(i)}=\hat{D}_{A}{ }^{B^{\prime}} \hat{D}_{B B^{\prime}} \bar{\beta}^{A}{ }_{(i)}=0 .
$$

If $\alpha^{A}{ }_{(i)}$ and $\bar{\beta}^{A}{ }_{(i)}$ are smooth then they satisfy the above equation in its strong form. Now, we turn to the question whether $\alpha^{A}{ }_{(i)}$ and $\bar{\beta}_{(i)}^{A}$ satisfy the first order elliptic equations (31). It can be shown by supposing a contradiction [9], i.e. we assume there are such $\bar{\lambda}_{B^{\prime}}=\hat{D}_{B B^{\prime}} \alpha^{B}{ }_{(i)} \neq 0$ and $\bar{\gamma}_{B^{\prime}}=\hat{D}_{B B^{\prime}} \bar{\beta}^{B}{ }_{(i)} \neq 0$. Substituting them into relation (24) we obtain the contradiction. All these conclude the proof of the existence theorem.

In order to study boundary conditions for $\alpha^{A}{ }_{(i)}$ and $\bar{\beta}_{A(i)}$ spinors we fix an asymptotically null hypersurface and choose a system of Bondi coordinates such that $\Sigma$ asymptotically approaches a null cone [20]. For a spinor basis $\left(o^{A}, i^{A}\right)$ we introduce a null tetrad $l^{a}, n^{a}, m^{a}, \bar{m}^{a}$ in such a way that $n^{a}=i^{A} \bar{i}^{A^{\prime}}$ and $l^{a}=o^{A} \bar{o}^{A^{\prime}}$ be the ingoing and outgoing null vectors orthogonal to $\Sigma$ and $l_{a} n^{a}=1$. The conjugate complex null vectors $m^{a}=o^{A} \bar{i}^{A^{\prime}}$ are normalized as follows: $m^{a} \bar{m}_{a}=-1$ and $o_{A} i^{A}=1$.

We expand $\alpha^{A(i)}$ and $\bar{\beta}_{A(i)}$ in terms of this basis, namely

$$
\begin{gathered}
\alpha^{A(i)}=X_{1}^{(i)} o^{A}+Y_{1}^{(i)} i^{A}, \\
\bar{\beta}^{A(i)}=X_{2}^{(i)} o^{A}+Y_{2}^{(i)} i^{A} .
\end{gathered}
$$

The two-surface bivector is $l^{[a} n^{b]} d S$, so the right-hand side of equation (24) may be written as

$$
\frac{1}{2} \int_{S} d S^{a b} \hat{\nabla}_{a} \xi_{b}=\frac{1}{2} \int_{S} d S\left(n_{a} D \xi^{a}-l_{a} \Delta \xi^{a}\right),
$$


where $D=l^{a} \nabla_{a}$ and $\Delta=n^{a} \nabla_{a}$. The Weyl equations may be expressed in the form

$$
\begin{aligned}
& i_{A} D \alpha_{(i)}^{A}+\frac{i}{2} e^{2 \phi} i_{A} D a \alpha_{(i)}^{A}-o_{A} \bar{\delta} \alpha_{(i)}^{A}-\frac{i}{2} e^{2 \phi} o_{A} \bar{\delta} a \alpha_{(i)}^{A}=0, \\
& o_{A} \Delta \alpha_{(i)}^{A}+\frac{i}{2} e^{2 \phi} o_{A} \Delta a \alpha_{(i)}^{A}-i_{A} \delta \alpha_{(i)}^{A}-\frac{i}{2} e^{2 \phi} i_{A} \delta a \alpha_{(i)}^{A}=0, \\
& \bar{i}_{A^{\prime}} D \beta^{A^{\prime}}{ }_{(i)}+\frac{i}{2} e^{2 \phi} \bar{i}_{A^{\prime}} D a \beta^{A^{\prime}}{ }_{(i)}-\bar{o}_{A^{\prime}} \delta \beta^{A^{\prime}}{ }_{(i)}-\frac{i}{2} e^{2 \phi} \bar{o}_{A^{\prime}} \delta a \beta^{A^{\prime}}{ }_{(i)}=0 \text {, } \\
& \bar{o}_{A^{\prime}} \Delta \beta^{A^{\prime}}{ }_{(i)}+\frac{i}{2} e^{2 \phi} \bar{o}_{A^{\prime}} \Delta a \beta^{A^{\prime}}{ }_{(i)}-\bar{i}_{A^{\prime}} \bar{\delta} \beta^{A^{\prime}}{ }_{(i)}-\frac{i}{2} e^{2 \phi} \bar{i}_{A^{\prime}} \bar{\delta} a \beta^{A^{\prime}}{ }_{(i)}=0,
\end{aligned}
$$

where $\delta=m^{a} \nabla_{a}$ and $\bar{\delta}=\bar{m}^{a} \nabla_{a}$.

By virtue of (40) and the above Weyl equations the boundary term on $S$ imply

$$
\begin{aligned}
\frac{1}{2} \int_{S} d S^{a b} \hat{\nabla}_{a} \xi_{b} & =\frac{1}{2} \int_{S} d S\left[\rho X_{1} \bar{X}_{1}+\mu Y_{1} \bar{Y}_{1}+2 \operatorname{Re} \bar{Y}_{1}\left(\delta X_{1}+\beta X_{1}\right)\right] \\
& +\frac{1}{2} \int_{S} d S\left[\rho X_{2} \bar{X}_{2}+\mu Y_{2} \bar{Y}_{2}+2 R e \bar{Y}_{2}\left(\delta X_{2}+\beta X_{2}\right)\right] \\
& +\frac{i}{2} \int_{S} d S e^{2 \phi} \delta a\left(X_{1} \bar{Y}_{1}+X_{2} \bar{Y}_{2}\right)+\text { complex conjugate } \\
& +\sqrt{2} \int_{S} d S\left[e^{-\phi} \phi_{1}\left(\bar{\alpha}^{A^{\prime}(i)} \beta_{A^{\prime}(i)}\right)+e^{-\phi} \bar{\phi}_{1}\left(\alpha^{A(i)} \bar{\beta}_{A(i)}\right)\right]
\end{aligned}
$$

where the spin coefficients are $\rho=o^{A} \bar{\delta} o_{A}, \mu=i^{A} \delta i_{A}, \beta=i^{A} \delta o_{A}$ and a complex scalar costructed from a Maxwell spinor $\phi_{A B}$ is defined as

$$
\phi_{1}=\phi_{A B} o^{A} i^{B}
$$

By the definition, $\rho=0$ on a future apparent horizon. We impose the following boundary conditions on $S$ when it is a future apparent horizon [23]

$$
\alpha^{A(i)} o_{A}=\beta^{A^{\prime}(i)} \bar{o}_{A^{\prime}}=0
$$

On the other hand, on a past apparent horizon $\mu=0$ and one can input the boundary conditions [23] of the form:

$$
i^{A} \alpha_{A(i)}=\bar{i}^{A^{\prime}} \beta_{A^{\prime}(i)}=0 .
$$

If the condition (47) for a future apparent horizon or (48) for a past apparent horizon are satisfied, then the whole surface term given by (45) disappears.

To prove the existence theorem in the case of inner boundaries of $\Sigma$ we proceed similary to the case described above. Namely, one proves the existence of solutions of equations (37) with the adequate boundary conditions. First we shall consider the case when $S$ is a future apparent horizon. From relation (47), using (38) one finds that

$$
\left.Y_{1}^{(i)}\right|_{S}=\left.Y_{2}^{(i)}\right|_{S}=0 .
$$


The additional boundary conditions are as follows:

$$
\left.\hat{D}^{1^{\prime}}{ }_{B} \alpha^{B}{ }_{(i)}\right|_{S}=\left.\hat{D}^{1^{\prime}}{ }_{B} \bar{\beta}^{B}{ }_{(i)}\right|_{S}=0 .
$$

Proceeding as before we show the existence of $a^{A}{ }_{(i)}$ and $b^{A}(i) \in \mathcal{H}$ such that $\alpha^{A}{ }_{(i)}$ and $\bar{\beta}^{A}{ }_{(i)}$ given by the relations (49) satisfy (37). Since $\alpha_{\infty(i)}^{A}$ and $\bar{\beta}_{\infty(i)}^{A}$ are chosen to vanish in the neighbourhood of $S$, then it turns out that $a^{A}{ }_{(i)}$ and $b_{(i)}^{A}$ assert the boundary conditions (49) and (50). It can be seen considering equation (36), integrating them twice by parts and using boundary conditions (49) and the fact that $\sigma^{A} \in C_{0}^{\infty} \cap \mathcal{H}$, and $\sigma^{0^{\prime}}$ can take arbitrary values on $S$.

The extension of the proof to the case when $S$ is past apparent horizon is straightforward. From (39) and (48) one obtains the boundary conditions

$$
\left.X_{1}^{(i)}\right|_{S}=\left.X_{2}^{(i)}\right|_{S}=0
$$

and the suplementary boundary conditions on a future apparent horizon yields

$$
\left.\hat{D}^{0^{\prime}}{ }_{B} \alpha^{B}{ }_{(i)}\right|_{S}=\left.\hat{D}^{0^{\prime}}{ }_{B} \bar{\beta}^{B}{ }_{(i)}\right|_{S}=0 \text {. }
$$

The rest of the proof is the same as in the case of the future apparent horizon.

To complete our considerations we shall cosider the boundary terms at infinity. If $\alpha^{A}{ }_{(i)}$ and $\bar{\beta}_{A(i)}$ approach the supercovariantly constant spinor at infinity, then having in mind the attitude presented in [2] it follows that

$$
\frac{1}{2} \int_{S^{\infty}} d S^{a b} \hat{\nabla}_{a} \xi_{b}=\frac{1}{4} P_{m} \xi_{\infty}^{m}+\frac{1}{\sqrt{2}} Q_{(F-\phi)} R e\left(\alpha_{\infty}^{A(i)} \bar{\beta}_{\infty A(i)}\right)+\frac{1}{\sqrt{2}} P_{(F-\phi)} \operatorname{Im}\left(\alpha_{\infty}^{A(i)} \bar{\beta}_{\infty A(i)}\right)
$$

where the dilaton-electric charge and dilaton-magnetic charge are defined respectively as

$$
Q_{(F-\phi)}=2 \int_{S^{\infty}} d S e^{-\phi_{\infty}} \operatorname{Re} \phi_{1}, \quad P_{(F-\phi)}=2 \int_{S^{\infty}} d S e^{-\phi_{\infty}} \operatorname{Im} \phi_{1} .
$$

Inspection of equation (24) reveals that the Bondi mass $M_{B O N D I}=\sqrt{P_{m} P^{m}}$ must be positive for all $\alpha_{\infty}^{A(i)}$ and $\bar{\beta}_{\infty A(i)}$, hence

$$
P_{m} P^{m} \geq Q_{(F-\phi)}^{2}+P_{(F-\phi)}^{2}
$$

\section{Conclusions}

In summary, we have studied the positivity of the Bondi mass in the low-energy string theory, the so-called EMAD gravity. We considered a non-singular spacelike hypersurface which 
asymptotically approached a null cone. One assumes that the conditions for energy-momentum tensor (28) and the specific relations amongs the fields in the theory (27) are satisfied. Moreover, the entire system is invariant under the supersymmetry transformations. Then the Bondi four-momentum is a future directed timelike vector. The square of the Bondi mass is greater than or equal to the sum of the squares of the total dilaton-electric or dilaton-magnetic charges.

The generalization of the above mentioned proof to the case when the hypersurface has inner boundaries was also provided. We considered the case of a future and past apparent horizons establishing the adequate boundary conditions for two-component spinors on them. One concludes, that if the above mentioned conditions for energy momentum tensor and fields in EMAD gravity are satisfied then the square of the Bondi mass of the spacetime containing black hole was greater or equal to the sum of the squares of the total dilaton-electric and dilatonmagnetic charges of the black hole.

\section{Acknowledgment}

I would like to thank the unknown referees for very useful comments. 


\section{References}

[1] Nester J and Israel W 1981 Phys.Lett.A 85259.

[2] Witten E 1981 Commun.Math.Phys. 80381.

[3] Nester J 1981 Phys.Lett.A 83241.

[4] Horowitz G and Perry M 1982 Phys.Rev.Lett. 48371.

[5] Ashtekar A and Horowitz G 1982 Phys.Lett.A 89181.

[6] Schoen R and Yau S-T 1979 Commun.Math.Phys. 6545.

[7] Schoen R and Yau S-T 1982 Phys.Rev.Lett. 48369.

[8] Ludvigsen M and Vickers J 1981 J.Phys.A 14 L389.

[9] Reula O and Tod K.P 1984 J.Math.Phys. 251004.

[10] Horowitz G and Tod K.P 1982 Commun.Math.Phys. 85429.

[11] Bergquist G 1992 Class.Quantum Grav. 9 1917, Bergquist G 1993 Phys.Rev.D 48628.

[12] Rogatko M 1995 Class.Quantum Grav. 12 3115, Rogatko M 2000 Class.Quantum Grav. 1711.

[13] Penrose R, Rindler W 1985 Spinors and Space-time vol.I (Cambridge: Cambridge University Press).

[14] Kallosh R, Linde A, Ortin T, Peet A and van Proyen A 1992 Phys.Rev.D 465278.

[15] Cremmer E and Scherk J 1978 Phys.Lett.B 7461.

[16] Gibbons G.W, Hull C.M and Warner N.P 1983 Nucl.Phys.B 218 173, Gibbons G.W, Kastor D, London L.A.J, Townsend P.K, and Trashen J 1994 Nucl.Phys.B 416850.

[17] Gibbons G.W and Hull C.M 1982 Phys.Lett 109B 190.

[18] Reula O 1982 J.Math.Phys. 23810.

[19] Rudin W 1973 Functional Analysis (New York: McGraw-Hill). 
[20] Bondi H, van der Burg M.G.J and Metzner A.W.K 1962 Proc.R.Soc. A269 21.

[21] Exton A, Newman E.T, and Penrose R 1969 J.Math.Phys. 7863.

[22] Bramson B.D 1975 Proc.R.Soc A341 451.

[23] Gibbons G.W, Hawking S.W, Horowitz G.T and Perry M.J 1983 Commun.Math.Phys. 88 295. 